\title{
Sintomas osteomusculares e desempenho ocupacional: um estudo entre músicos instrumentistas no contexto do Distrito Federal, Brasil*
}

\section{Musculoskeletal symptoms and occupational performance: a study among instrumentalist musicians in the Federal District, Brazil}

\author{
Andréia Louise A. de Carvalho ${ }^{1}$, Tatiana Barcelos Pontes ${ }^{2}$, Lilian Dias B. Massa ${ }^{3}$, \\ Daniela da Silva Rodrigues ${ }^{4}$, Pedro H.T.Q. de Almeida ${ }^{5}$
}

http://dx.doi.org/10.11606/issn.2238-6149.v27i2p165-171

De Carvalho ALA, Pontes TB, Massa LDB, Rodrigues DS, Almeida PHTQ. Sintomas osteomusculares e desempenho ocupacional: um estudo entre músicos instrumentistas no contexto do Distrito Federal, Brasil. Rev Ter Ocup Univ São Paulo. 2016 maio-ago.;27(2):165-71.

\begin{abstract}
RESUMO: Objetivo: Determinar a prevalência de sintomatologia osteomuscular entre músicos de uma universidade federal, verificando seu impacto sobre a participação em atividades de vida diária e o desempenho de atividades laborais. Método: 26 músicos foram avaliados por meio do Questionário de Disfunção do Ombro, Braço e Mão e Questionário Nórdico de Sintomas Osteomusculares. A relação entre sintomatologia musculoesquelética e desempenho ocupacional foi avaliada por meio dos testes de Mann-Whitney, Teste de Correlação de Spearman e Teste de Chi-Quadrado. Resultados: Foi observado predomínio de queixas osteomusculares nas regiões dos membros superiores e tronco, com baixa procura dos músicos por tratamentos de saúde. Observaram-se diferenças significativas no desempenho de atividades de vida diária e realização de atividades relacionadas à prática musical entre participantes que apresentavam queixas osteomusculares em membros superiores. Conclusão: $\mathrm{O}$ desempenho da atividade musical pode levar a processos de adoecimento, influenciando as atividades laborais, e outras áreas do desempenho ocupacional. Ações voltadas para a prevenção e tratamento de queixas osteomusculares são importantes para a elaboração de estratégias que visam preservar e promover a saúde desta população.
\end{abstract}

DESCRITORES: Terapia ocupacional; Dor musculoesquelética; Músico; Doenças ocupacionais.
De Carvalho ALA, Pontes TB, Massa LDB, Rodrigues DS, Almeida PHTQ. Musculoskeletal symptoms and occupational performance: a study among instrumentalist musicians in the Federal District, Brazil. Rev Ter Ocup Univ São Paulo. 2016 May-Aug.;27(2):165-71.

ABSTRACT: Objective: To determine the prevalence of musculoskeletal symptomatology among musicians from a federal university, verifying its impact on daily activities and work performance. Methods: 26 musicians have been assessed using the Disabilities of the Arm, Shoulder, and Hand Questionnaire and the Nordic Musculoskeletal Questionnaire. Relation between musculoskeletal symptomatology and occupational performance was evaluated by Mann-Whitney test, Spearman's rank correlation coefficient, and Chi-square test. Results: We observed preponderance of musculoskeletal complaints in the upper limbs and torso regions, with low demand for medical services by musicians. There was significant difference in the performance of daily activities and music practice among participants who had musculoskeletal complaints in upper limbs. Conclusion: Playing-related musculoskeletal disorders may affect work activities and other areas of occupational performance. Initiatives on prevention and treatment of musculoskeletal disorders are important to the development of strategies aiming at preserving and promoting this population health.

KEYWORDS: Occupational therapy; Musculoskeletal pain; Music; Occupational diseases.

*Este trabalho é parte do Trabalho de Conclusão de Curso em terapia ocupacional intitulado Saúde do Músico: Sintomas Osteomusculares e Perfil Ocupacional. $\mathrm{O}$ trabalho não foi apresentado em nenhum evento anteriormente à sua submissão.

1. Terapeuta Ocupacional, Secretaria de Saúde do Distrito Federal. E-mail: deialac@gmail.com

2. Curso de Terapia Ocupacional da Universidade de Brasília-UnB. E-mail: tatianapontes@unb.br

3. Curso de Terapia Ocupacional do Instituto Federal de Educação, Ciência e Tecnologia do Rio de Janeiro - IFRJ. E-mail: lilian.dias@gmail.com

4. Curso de Terapia Ocupacional da Universidade de Brasília - UnB. E-mail: danirodrigues.to@gmail.com

5. Curso de Terapia Ocupacional da Universidade de Brasília - UnB. E-mail: pedroalmeida.to@gmail.com

Endereço para correspondência: Pedro H.T.Q. de Almeida - Curso de Terapia Ocupacional da Universidade de Brasília - Faculdade de Ceilândia. Centro Metropolitano, conjunto A, lote 01, Brasília - DF. CEP: 72220-900. 


\section{INTRODUÇÃO}

$\mathrm{T}$ ocar um instrumento musical de forma profissional exige do músico um grande nível de atividade, tanto mental quanto física e inclui uma complexa interação entre fatores relacionados ao controle motor, musicalidade e expressividade ${ }^{1}$. Embora a ocorrência de alterações decorrentes da exposição a elevados níveis de ruído sonoro seja o principal objeto de pesquisas na área ${ }^{2}$, considerável número de estudos ao longo da última década tem focado a elevada prevalência de distúrbios osteomusculares observada entre músicos instrumentistas ${ }^{1}$.

Em estudo realizado junto a população holandesa, Kok et al. ${ }^{3}$ observaram a ocorrência de sintomas osteomusculares em $89 \%$ de uma amostra composta por 83 musicistas, similar ao descrito por estudos conduzidos em países europeus ${ }^{4-6}$, África do $\mathrm{Sul}^{7}$ e Austrália ${ }^{8}$. Observa-se ainda que o desenvolvimento de queixas osteomusculares é duas vezes mais frequente entre músicos quando comparados a população geral ${ }^{3}$.

Diversos fatores são elencados como agravantes para a saúde deste grupo profissional: a elevada exigência que o instrumento impõe ao corpo, incluindo alterações posturais impostas durante a prática musical e o transporte do mesmo $^{3,9}$; o início precoce da atividade, geralmente entre infância e adolescência ${ }^{5}$; extenso período de prática musical e o acúmulo de funções do instrumentista dentro de uma orquestra sinfônica ou banda ${ }^{10}$.

Estudos sugerem que os principais problemas observados entre músicos estão relacionados aos membros superiores, pescoço e boca ${ }^{1,3,11}$. Dentre os quadros sintomáticos relacionados a alterações no membro superior, as tendinopatias, mialgias e a síndrome do superuso (overuse) são as condições mais comuns entre esta população ${ }^{4}$. As queixas no aparelho motor manifestamse muitas vezes como dor, fraqueza ou aumento de tensão muscular ${ }^{12}$, sendo observada prevalência de distúrbios musculoesqueléticos entre 29 a $90 \%$ dos casos ${ }^{1}$.

A presença de dor e demais queixas osteomusculares afeta diretamente o desempenho ocupacional destes profissionais, tanto para a realização de atividades do cotidiano quanto para o exercício das suas atividades profissionais. Paarup etal. ${ }^{13}$. Concomitantemente,Ackermann et al. ${ }^{8}$ observaram que $84 \%$ de 377 músicos australianos apresentaram incapacidade para exercer atividades laborais em decorrência de dor ou lesões osteomusculares ${ }^{8}$.

Observa-se que a incidência de sintomas de dor musculoesquelética entre músicos pode ser percebida desde a infância: Nawrocka et al. ${ }^{5}$ descrevem a presença de dor em membros superiores em aproximadamente $44 \%$ de estudantes de música entre 10 e 18 anos, sendo crescente o aumento da incidência de queixas osteomusculares a cada ano consecutivo de prática 5 .

Existem poucos trabalhos publicados sobre a saúde do músico no Brasil, com foco sobre o impacto de sintomas osteomusculares ${ }^{12,14-16}$, sendo mais escassas ainda pesquisas sobre estudantes de música no país ${ }^{16}$.

Os exames constantes, altas expectativas e pressão por parte dos pais e professores, assim como as competições artísticas obrigam os estudantes à excessiva prática sem supervisão ${ }^{5,15}$. Tal fato é potencialmente alarmante, pois durante a supervisão os erros técnicos são corrigidos, evitando a sobrecarga do sistema musculoesquelético dos jovens músicos ${ }^{7}$. Além da participação em atividades acadêmicas, é comum a atuação de estudantes de música em atividades laborais, como apresentações artísticas e aulas particulares, o que contribui ainda mais para a sobrecarga desta população ${ }^{15}$.

Tendo em vista o exposto, o objetivo desta pesquisa foi (a) obter um perfil da prevalência de sintomatologia osteomuscular entre músicos instrumentistas de uma universidade federal; (b) verificar a associação entre a sintomatologia osteomuscular e alteração na participação em atividades; (c) verificar a associação entre a sintomatologia osteomuscular e alteração no desempenho dos músicos em sua atividade profissional.

\section{METODOLOGIA}

Trata-se de um estudo transversal, descritivo e exploratório, de abordagem quantitativa. Foi realizado através de uma parceria entre o serviço de terapia ocupacional de um hospital universitário e o departamento de música de uma universidade pública, durante os meses de outubro a dezembro de 2014.

Foram convidados a participar da pesquisa 70 músicos vinculados ao departamento, como alunos de graduação em música, mestrandos e doutorandos. Os convites foram realizados por intermédio dos professores responsáveis pelas disciplinas frequentadas pelos estudantes e através de e-mail e contato pessoal com os estudantes.

A pesquisa teve como critérios de inclusão: ser músico instrumentista, possuir vínculo com o curso de música da instituição determinada; possuir idade maior que 18 anos. Foram excluídos participantes que apresentassem problemas neurológicos ou ortopédicos não relacionados à atividade musical, como acidente vascular encefálico, 
traumatismos decorrentes de acidentes de trânsito, quedas e outras doenças correlatas.

Os participantes foram orientados a responder um formulário autoaplicável contendo uma ficha de anamnese, elaborada pelos autores, com questões referentes a informações demográficas e à atividade de músico instrumentista. Além da ficha inicial, os participantes responderam o Questionário de Disfunção do Ombro, Braço e Mão (DASH) e o Questionário Nórdico de Sintomas Osteomusculares (QNSO).

Para a elaboração da ficha de anamnese foram selecionadas questões referentes a variáveis sociodemográficas, de perfil ocupacional e condições de saúde, objetivando descrever o perfil da população estudada e a relação destas variáveis sobre a incidência de dor e disfunção decorrente da prática musical. Idade, sexo, atividades profissionais realizadas, horas de estudo, posição ao tocar, transporte do instrumento, conhecimento sobre saúde do músico e estratégias de preservação da saúde foram informações coletadas através deste instrumento.

\section{O Nordic Musculoskeletal Questionnaire} (Questionário Nórdico de Sintomas Osteomusculares QNSO) ${ }^{17}$ foi elaborado com o intuito de padronizar a mensuração do relato de sintomas e queixas osteomusculares e, assim, facilitar a comparação dos resultados entre os estudos. O questionário define como sintomas e queixas osteomusculares a presença de dor, formigamento e/ ou dormência nas regiões do pescoço, ombros, costas, cotovelos, punhos e mãos, quadril, joelhos e tornozelos. Há três formas do QNSO: uma forma geral, compreendendo todas as áreas anatômicas, e outras duas específicas para as regiões lombares, de pescoço e ombros ${ }^{18}$. Em todas as formas do questionário, as queixas apresentadas pelo paciente referentes a dois momentos, 12 meses e sete dias anteriores ao preenchimento do QNSO. Além das questões sobre queixas osteomusculares, o QNSO apresenta duas questões referentes a procura por auxílio profissional devido a queixa apresentada e limitações em atividades de autocuidado, de trabalho e lazer ocasionadas pela queixa ou sintoma osteomuscular. Para o presente estudo, a forma geral do QNSO foi utilizada e as quatro questões apresentadas respondidas.

O DASH é um questionário autoaplicável que avalia o impacto de disfunções do membro superior sobre a realização de atividades de vida diária e atividades profissionais desempenhadas pelo respondente ${ }^{19}$. Possui 30 questões com intuito de mensurar a dificuldade enfrentada durante o desempenho de atividades cotidianas (DASH-1), compreendendo 21 questões voltadas a realização tarefas, seis itens referentes a intensidade de sintomas e três itens compreendendo participação em atividades sociais.
O questionário conta com dois módulos opcionais, que avaliam o impacto da disfunção dos membros superiores sobre o desempenho de atividades vinculadas à prática musical ou esportiva (DASH-2) e atividades laborais (DASH-3). O escore da avaliação é apresentado em uma escala positiva entre 0 e 100 pontos, com resultados maiores indicando melhor desempenho funcional. Pelo fato dos módulos opcionais apresentarem as mesmas questões e a prática profissional dos participantes do presente estudo basear-se exclusivamente na prática musical, foram considerados os resultados obtidos por meio dos questionários DASH-1 e DASH-2.

Os dados referentes a fatores sociodemográficos foram analisados por meio de estatística descritiva. Diferenças entre variáveis categóricas medidas pelo QNSO foram analisadas por meio do teste de Chi-Quadrado e Teste Exato de Fisher.

Os participantes foram divididos em dois grupos, de acordo com a presença de dor em membros superiores, determinada através do QNSO, a fim de verificar a associação entre dor e restrições na participação de atividades, mensurada pelo DASH. Uma vez que os escores obtidos não apresentaram distribuição normal de acordo com o teste de Shapiro-Wilk, a comparação entre os grupos foi realizada por meio do teste não paramétrico de Mann-Whitney. Por fim, os dados referentes à idade dos participantes, escores do DASH e horas de prática musical semanal foram analisados por meio do teste de correlação de Spearman.

Para todas as análises considerou-se um nível de significância de $\mathrm{p}<0.05$. A análise estatística foi realizada através do software SPSS, versão 20.0 e o cálculo do poder estatístico por meio do software G-Power, versão 3.1.

Todos os músicos que aceitaram participar da pesquisa foram esclarecidos quanto ao projeto e os objetivos do estudo, assinando, posteriormente, o Termo de Consentimento Livre e Esclarecido - TCLE. O projeto foi aprovado pelo comitê de ética da instituição proponente (parecer 845.114/2014).

\section{RESULTADOS}

\section{Participantes}

Dos 70 participantes convidados, 26 (37.1\%) retornaram os formulários preenchidos. A amostra foi composta por 21 indivíduos do sexo masculino e apresentou idade média de 25,2 anos ( \pm 7,2 anos). A maioria dos respondentes foi composta por graduandos em música, com tempo médio de prática semanal de 9,1 horas 
( \pm 6,7 horas). Além de atividades acadêmicas, mais da metade dos participantes realizava apresentações musicais e ministrava aulas de música concomitante aos estudos.

No que se refere à compreensão do modo de trabalho e sua relação com o processo de saúde e doença, $57 \%$ dos participantes afirmam possuir conhecimentos prévios sobre a saúde do músico e $30 \%$ realizam atividades físicas como estratégia de preservação de sua saúde. Os dados demográficos são ilustrados na Tabela 1.

Tabela 1 - Dados demográficos

\begin{tabular}{l|c}
\hline \multicolumn{2}{l}{ n (\%) } \\
\hline Sexo & $5(19.2)$ \\
\hline Feminino & $21(80.8)$ \\
\hline Masculino & \\
\hline Formação & $24(92.3)$ \\
\hline Graduando & $1(3.8)$ \\
\hline Mestrando & $1(3.8)$ \\
\hline Doutorando & $23(88.5)$ \\
\hline Atividades Desempenhadas* & $18(69.2)$ \\
\hline Estudante & $13(50)$ \\
\hline Realiza apresentações musicais & $5(19.2)$ \\
\hline Dá aulas de música & $3(11.5)$ \\
\hline Participa de orquestra & $19(73.1)$ \\
\hline Outras atividades profissionais & $7(26.9)$ \\
\hline Posição ao Tocar & $8(30.8)$ \\
\hline Sentado & $15(57.7)$ \\
\hline Em Pé & \\
\hline Fatores Protetivos & \\
\hline Realiza Exercício Físico & \\
\hline $\begin{array}{l}\text { Possui conhecimentos sobre saúde do } \\
\text { músico }\end{array}$ & \\
\hline
\end{tabular}

*Questão de múltipla escolha: a porcentagem total pode ultrapassar $100 \%$

\section{Queixas osteomusculares e participação em atividades}

A avaliação pelo QNSO indicou predomínio de queixas osteomusculares na região lombar baixa $(53,8 \%)$, seguida por ombro $(46,2 \%)$, região lombar alta $(34,6 \%)$, pescoço $(34,6 \%)$, punho e mãos $(34,6 \%)$ (Tabela 2$)$. Os participantes apresentaram percentual de queixas similares nos recortes temporais propostos pelo QNSO (12 meses e 7 dias anteriores ao momento da avaliação), com exceção de queixas na região cervical, que diminuiram no segundo momento.
Tabela 2 - Queixas osteomusculares, interferência em atividades e procura por profissionais de saúde

\begin{tabular}{l|c|c|c|c}
\hline $\begin{array}{l}\text { Região } \\
\text { Corporal }\end{array}$ & $\begin{array}{c}\text { Queixa- } \\
\mathbf{1 2} \text { meses } \\
\mathbf{n}(\%)\end{array}$ & $\begin{array}{c}\text { Queixa-7 } \\
\text { dias } \\
\text { n (\%)* }\end{array}$ & $\begin{array}{c}\text { Interferência } \\
\text { em } \\
\text { Atividades } \\
\text { n (\%)* }\end{array}$ & $\begin{array}{c}\text { Procura por } \\
\text { Profissional } \\
\text { de Saúde } \\
\text { n (\%)* }\end{array}$ \\
\hline $\begin{array}{l}\text { Pescoço } \\
\text { Região } \\
\text { Lombar } \\
\text { Alta }\end{array}$ & $9(34.6)$ & $2(7.7)$ & $4(15.4)$ & $1(3.8)$ \\
\hline Ombro & $12(46.2)$ & $6(23.1)$ & $5(19.2)$ & $3(11.5)$ \\
\hline $\begin{array}{l}\text { Cotovelo } \\
\text { Punho e } \\
\text { Mãos }\end{array}$ & $3(11.5)$ & $1(3.8)$ & $1(3.8)$ & $1(3.8)$ \\
\hline $\begin{array}{l}\text { Região } \\
\text { Lombar } \\
\text { Baixa }\end{array}$ & $14(53.8)$ & $10(38.5)$ & $7(26.9)$ & $6(23.1)$ \\
\hline Quadris & $3(11.5)$ & $1(3.8)$ & $0(0)$ & $0(0)$ \\
\hline Joelhos & $7(26.9)$ & $5(19.2)$ & $2(7.7)$ & $0(0)$ \\
\hline Pés & $6(23.1)$ & $3(11.5)$ & $2(7.7)$ & $1(3.8)$ \\
\hline
\end{tabular}

*Questão de múltipla escolha: a porcentagem total pode ultrapassar 100\%

A comparação pelo teste de exato de Fisher e análise post-hoc pelo residual ajustado do teste de ChiQuadrado indicaram associações significativas entre os quadros de queixas osteomuculares em 12 meses e 7 dias anteriores à avaliação para as regiões lombar superior $(p=0.002)$, punho e mãos $(p=0.012)$, lombar inferior $(\mathrm{p}<0.001)$, joelho e pés $(\mathrm{p}=0.01)$, sugerindo a permanência de queixas entre os participantes por períodos de até um ano.

A presença de queixas osteomusculares apresentou interferência significativa sobre a realização de atividades pelos participantes. Embora o QNSO não especifique quais atividades foram influenciadas, associações significativas entre queixas musculoesqueléticas e prejuízo no desempenho de atividades foram observadas para as regiões do pescoço $(\mathrm{p}=0.008)$, região lombar alta $(\mathrm{p}=0.03)$, ombro $(\mathrm{p}=0.01)$, punho e mãos $(0.03)$, região lombar baixa (0.006) e pés (0.04).

Apesar da limitação para o desenvolvimento de atividades devido às queixas musculoesqueléticas, a procura por profissionais de saúde nos 12 meses anteriores a avaliação foi consideravelmente baixa entre os participantes, sendo significativa apenas para queixas relacionadas à região lombar baixa $(\mathrm{p}=0.01)$. Apesar do elevado número de queixas nas regiões do membro superior, apenas 9 participantes (34.6\%) procuraram profissionais de saúde, sendo que nenhum dos 
respondentes procurou auxílio profissional para problemas do punho e mãos.

O conhecimento prévio sobre saúde do músico, prática de atividade física regular e o fato de carregar o próprio instrumento musical não apresentaram diferenças significativas quanto a prevalência de queixas osteomusculares entre a população estudada, tanto nos últimos 12 meses quanto nos 7 dias anteriores a coleta de dados.

\section{Queixas osteomusculares e desempenho ocupacional}

Os participantes foram divididos em dois grupos de acordo com a presença de queixas osteomusculares em membros superiores nos 7 dias que antecederam a avaliação. Treze indivíduos ( $50 \%$ da amostra) apresentaram queixas em membros superiores.

A análise dos escores do DASH através do teste de Mann-Whitney indicou diferenças significativas entre os participantes que apresentavam queixas osteomusculares em membros superiores (Grupo 1-MMSS) quando comparados a indivíduos sem queixas nesta região (Grupo 2-Sem queixas). Foram observadas diferenças significativas quanto a realização de atividades de vida diária (DASH$1 ; p=0.04)$ e também para o desempenho de atividades relacionadas à prática musical (DASH-2; $\mathrm{p}=0.02)$ entre os participantes alocados nos dois grupos (Tabela 3 ).

Não foram observadas correlações significativas entre a idade, horas de prática por semana e o desempenho de atividades entre os participantes (Tabela 4).

Tabela 3 - Impacto funcional de queixas osteomusculares em membros superiores

\begin{tabular}{l|c|c|c|c}
\hline & $\begin{array}{l}\text { Grupo 1 - } \\
\text { MMSS } \\
\text { Média } \\
\text { (Desv.Pad) }\end{array}$ & $\begin{array}{l}\text { Grupo 2 - } \\
\text { Sem queixas } \\
\text { Média } \\
\text { (Desv.Pad) }\end{array}$ & p* & $\begin{array}{l}\text { Poder } \\
\text { Estatístico }\end{array}$ \\
\hline DASH-1 & $14.3(12.7)$ & $5.01(6.2)$ & 0.04 & $60 \%$ \\
\hline DASH-2 & $33.9(24.2)$ & $12.5(13.2)$ & 0.02 & $74 \%$ \\
\hline
\end{tabular}

*Teste de Mann-Whitney

Tabela 4 - Coeficiente de Correlação de Spearman para as variáveis Idade, Horas de Prática e escore do DASH

\begin{tabular}{l|c|c|c|c}
\hline & Idade & $\begin{array}{c}\text { Horas de } \\
\text { Prática }\end{array}$ & DASH-1 & DASH-2 \\
\hline Idade & - & -0.22 & 0.36 & 0.02 \\
\hline Horas de Prática & & - & 0.22 & -0.14 \\
\hline DASH-1 & & & - & $0.71^{*}$ \\
\hline DASH-2 & & & & - \\
\hline
\end{tabular}

$* \mathrm{p}<0.01$

\section{DISCUSSÃO}

Os resultados obtidos no presente estudo indicaram maior distribuição de queixas osteomusculares nas regiões do membro superior e tronco. Os dados corroboram resultados obtidos em outros estudos. Nowrocka et al. ${ }^{5}$ avaliaram a prevalência e intensidade de dor osteomuscular em 225 estudantes de música poloneses entre 10 e 18 anos de idade e observaram distribuição semelhante ao encontrado no presente estudo: maior incidência de dor no pescoço $(60,4 \%)$, punho $(44,4 \%)$, lombar alta $(41,7 \%)$ e lombar baixa $(38,2 \%)^{5}$. Steinmetz et al. $^{4}$ reportam que $72,8 \%$ dos participantes de um estudo realizado na Alemanha apresentaram dor em região cervical e torácica e mais da metade dos respondentes afirmou ter dor em membros superiores ${ }^{4}$. Resultados similares são observados em outros estudos com músicos, nos quais a incidência de queixas relacionadas a dor osteomuscular é majoritária nas regiões de pescoço, tronco e membros superiores ${ }^{3,6,7,10,14}$.

Embora o diagnóstico de disfunções relacionadas ao uso excessivo dos membros superiores seja controverso ${ }^{20}$, o dano a estruturas anatômicas decorrente do estresse constante sobre músculos, tendões e ligamentos é a condição de mais alta prevalência entre músicos ${ }^{21}$.

A relação entre o sobreuso do membro superior e a alta incidência de desordens musculoesqueléticas entre músicos é o principal fator de risco biomecânico para esta classe profissional ${ }^{9}$, contribuindo com grande número de afastamentos e impedindo o desempenho de atividades laborais, muitas vezes de forma precoce 5 .

De acordo com a literatura, a maioria das lesões osteomusculares observadas entre músicos ocorre entre a terceira e quarta década de vida ${ }^{22}$. Em nosso estudo, apesar da média de idade dos participantes ser de 25,2 anos, metade dos músicos relatam já apresentar dor osteomuscular que comprometia o desempenho de atividades e o exercício de ocupações.

Indivíduos com queixas osteomusculares decorrentes do trabalho podem apresentar sintomas persistentes e dificuldades em desempenhar atividades de vida diária, desemprego, depressão e problemas familiares ${ }^{23}$. Neste estudo, a presença de queixas osteomusculares associou-se, significativamente, a prejuízo no desempenho de atividades de vida diária e nas atividades laborais.

Embora os resultados desse estudo indiquem a existência de impactos das queixas osteomusculares sobre o desempenho de atividades de vida diária, tal temática não é abordada pela literatura relacionada à saúde do músico. Apesar do crescente número de publicações referentes à investigação de sintomatologia musculoesquelética 
entre esta população, o foco destas pesquisas restringese as repercussões da dor ou limitação motora sobre o desempenho da atividade musical 1,4,5,9,21-24.

Em um estudo realizado em 15 países da Europa e Israel, dos 4839 respondentes que relataram dor crônica, $61 \%$ afirmou ser menos capaz ou incapaz de desempenhar atividades fora de casa, $19 \%$ perdeu o emprego e $13 \%$ precisou mudar de emprego em decorrência da dor. Neste mesmo estudo, um terço dos respondentes relatou não receber nenhum tipo de tratamento ${ }^{25}$, similar aos achados de Ioannou e Altenmuller ${ }^{24}$ no qual $35,5 \%$ dos estudantes de música não buscavam atendimento ${ }^{24}$. Tais dados contrastam com os resultados da presente pesquisa, na qual apenas $34,6 \%$ dos participantes procurou auxílio junto a profissionais de saúde nos 12 meses anteriores à coleta dos dados.

A prática de atividade física e conhecimentos sobre saúde do músico não influenciaram a incidência de queixas osteomusculares e não impactaram o desempenho de atividades entre os participantes. Tais resultados vão de encontro ao relatado por Lima et al ${ }^{14}$ que observaram a ineficiência de estratégias voltadas para a redução da exposição de músicos a fatores de risco ocupacionais, o que leva ao questionamento sobre a disponibilidade e eficácia destas medidas, sobretudo para a realidade brasileira $^{14}$.

Tendo em vista o exposto, este estudo se justifica pela escassez de estudos relacionados à ocorrência de sintomatologia osteomuscular entre músicos no Brasil e sobretudo o impacto destes sintomas sobre o desempenho das ocupações e atividades de vida diária nesta população, ressaltando a importância de maiores investigações sobre as estratégias e abordagens utilizadas para a prevenção e tratamento desta condição como tema para estudos futuros.

\section{REFERÊNCIAS}

1. Silva AG, La FM, Afreixo V. Pain prevalence in instrumental musicians: a systematic review. Med Probl Perform Art. 2015;30:8-19.

2. Raymond DM, Romeo JH, Kumke KV. A pilot study of occupational injury and illness experienced by classical musicians. Workplace Health Saf. 2012;60:19-24. DOI: 10.3928/21650799-20111227-01.

\section{Limitações do Estudo}

Apesar dos esforços despendidos pelos autores, a taxa de resposta obtida acarretou em uma reduzida amostra, o que inviabilizou o uso de técnicas de análise estatística inferencial, que possibilitassem a identificação de relações causais entre as múltiplas variáveis investigadas.

\section{CONCLUSÃO}

As demandas físicas e cognitivas necessárias para desempenhar a atividade musical podem levar ao processo de adoecimento desses trabalhadores. As queixas osteomusculares apresentadas nesse estudo são semelhantes a pesquisas prévias realizadas com músicos instrumentistas ${ }^{1,3-7,10,14,21}$. Apesar da alta prevalência de sintomatologia osteomuscular, observou-se pouca procura para o tratamento e adoção de estratégias para prevenção de queixas e sintomas osteomusculares entre a população pesquisada.

O entendimento das limitações motoras e do quadro álgico, que podem impactar no exercício de sua profissão ou de outras atividades do cotidiano, contribui para a elaboração de estratégias que visam preservar e promover a saúde dessa população estudada.

Pesquisas futuras voltadas para a compreensão da organização do trabalho, da percepção desse músico sobre as atividades que desempenha e sobre sua inserção dentro de um grupo podem levar a um maior entendimento dos agravos que acometem a saúde desses trabalhadores e das estratégias adotadas por eles para se manterem em atividade. Nesse panorama, com a colaboração dos músicos, é possível se pensar em ações que visam à diminuição dos fatores relacionados ao adoecimento e em estratégias que melhorem a qualidade de vida no trabalho.

3. Kok LM, Vlieland TP, Fiocco M, Nelissen RG. A comparative study on the prevalence of musculoskeletal complaints among musicians and non-musicians. BMC Musculoskelet Disord. 2013;14:9. DOI: 10.1186/1471-2474-14-9.

4. Steinmetz A, Scheffer I, Esmer E, Delank KS, Peroz I. Frequency, severity and predictors of playing-related musculoskeletal pain in professional orchestral musicians in Germany. Clin Rheumatol. 2015;34:965-73. DOI: 10.1007/ s10067-013-2470-5. 
5. Nawrocka A, Mynarski W, Powerska-Didkowska A, Grabara M and Garbaciak W. Musculoskeletal pain among Polish music school students. Med Probl Perform Art. 2014;29:64-9.

6. Fotiadis DG, Fotiadou EG, Kokaridas DG, Mylonas AC. Prevalence of musculoskeletal disorders in professional symphony orchestra musicians in Greece: a pilot study concerning age, gender, and instrument-specific results. Med Probl Perform Art. 2013;28:91-5.

7. Ajidahun AT, Phillips J. Prevalence of musculoskeletal disorders among instrumental musicians at a center for performing arts in South Africa. Med Probl Perform Art. 2013;28:96-9.

8. Ackermann B, Driscoll T, Kenny DT. Musculoskeletal pain and injury in professional orchestral musicians in Australia. Med Probl Perform Art. 2012;27:181-7.

9. Kaufman-Cohen Y, Ratzon NZ. Correlation between risk factors and musculoskeletal disorders among classical musicians. Occup Med (Lond). 2011;61:90-5. DOI: 10.1093/ occmed/kqq196.

10. Barbar AE, de Souza Crippa JA, de Lima Osorio F. Performance anxiety in Brazilian musicians: prevalence and association with psychopathology indicators. J Affect Disord. 2014;152-154:381-6. DOI: 10.1016/j.jad.2013.09.041.

11. Steinmetz A, Moller H, Seidel W, Rigotti T. Playing-related musculoskeletal disorders in music students-associated musculoskeletal signs. Eur J Phys Rehabil Med. 2012;48:625-33.

12. Frank A and CAv M. Queixas musculoesqueléticas em músicos: prevalência e fatores de risco. Rev Bras Reumatol. 2007;47:188-96.

13. Paarup HM, Baelum J, Holm JW, Manniche C, Wedderkopp N. Prevalence and consequences of musculoskeletal symptoms in symphony orchestra musicians vary by gender: a cross-sectional study. BMC Musculoskelet Disord. 2011; 12: 223. DOI: 10.1186/1471-2474-12-223.

14. Lima RC, Pinheiro TM, Dias EC and de Andrade EQ. Development and prevention of work related disorders in a sample of Brazilian violinists. Work. 2015;51:273-80. DOI: 10.3233/WOR-141904.

Recebido em: 30.10 .15

Aceito em: 13.01 .16
15. Valverde Alves C. Padrões físicos inadequados na performance musical de estudantes de violino. PER MUSI Rev Acad Musica. 2012;(26):128-39. DOI: http://dx.doi. org/10.1590/S1517-75992012000200013.

16. de Oliveira CFC, Vezzá FMG. A saúde dos músicos: dor na prática profissional de músicos de orquestra no ABCD Paulista RBSO. 2010;35:33-40. DOI: http://dx.doi. org/10.1590/S0303-76572010000100005.

17. Kuorinka I, Jonsson B, Kilbom A, et al. Standardised Nordic questionnaires for the analysis of musculoskeletal symptoms. Appl Ergon. 1987;18:233-7.

18. Pinheiro FA, Tróccoli BT, Carvalho Cd. Validação do Questionário Nórdico de Sintomas Osteomusculares como medida de morbidade. Rev Saúde Pública. 2002;36:307-12. DOI: http://dx.doi.org/10.1590/S0034-89102002000300008.

19. Orfale A, Araújo P, Ferraz M, Natour J. Translation into Brazilian Portuguese, cultural adaptation and evaluation of the reliability of the Disabilities of the Arm, Shoulder and Hand Questionnaire. Braz J Med Biol Res. 2005;38:2. DOI: http://dx.doi.org/10.1590/S0100-879X2005000200018.

20. Bird HA. Overuse syndrome in musicians. Clin Rheumatol. 2013;32:475-9. DOI:10.1007/s10067-013-2198-2.

21. Lee HS, Park HY, Yoon JO, et al. Musicians' medicine: musculoskeletal problems in string players. Clin Orthop Surg. 2013;5:155-60. DOI: 10.4055/cios.2013.5.3.155.

22. Sheibani-Rad S, Wolfe S and Jupiter J. Hand disorders in musicians: the orthopaedic surgeon's role. Bone Joint J. 2013;95-b:146-50. DOI: 10.1302/0301-620X.95B2.30092.

23. Spreeuwers D, de Boer AG, Verbeek JH, et al. Workrelated upper extremity disorders: one-year follow-up in an occupational diseases registry. Int Arch Occup Environ Health. 2011;84:789-96. DOI: 10.1007/s00420-011-0611-1.

24. Ioannou CI and Altenmuller E. Approaches to and Treatment Strategies for Playing-Related Pain Problems Among Czech Instrumental Music Students: An Epidemiological Study. Med Probl Perform Art. 2015;30:135-42.

25. Breivik H, Collett B, Ventafridda V, Cohen R, Gallacher D. Survey of chronic pain in Europe: prevalence, impact on daily life, and treatment. Eur J Pain. 2006;10(4):287-333. DOI: 10.1016/j.ejpain.2005.06.009. 